\title{
Extraskeletal Osteoma of the Eyelid-A Rare Case Report
}

\author{
Sudhir Singh, MS
}

Senior Consultant and HOD, Department of Ophthalmology, JW Global Hospital \& Research Centre, Mount Abu, Rajasthan, India

\begin{abstract}
Osteoma is a benign osteogenic lesion with a very slow growth, characterized by proliferation of either cancellous or compact bone. Depending on its location, osteoma may be classified as central, peripheral, or extraskeletal type. Osteoma has been reported in relation to the paranasal sinuses and less frequently with the orbit. We report a case of a 25-year-old female who developed extraskeletal osteoma on her left upper eyelid, without involvement of the paranasal sinuses or orbit. Extraskeletal osteoma of the eyelid is an extremely rare variety. The mass was surgically removed and sent for histopathologic examination, which showed osteoma. To the best of our knowledge, this is the first case of extraskeletal osteoma of the eyelid reported in the English language literature.
\end{abstract}

\section{Keywords}

Extraskeletal osteoma, lid osteoma, Sudhir Singh

Disclosure: Sudhir Singh, MS, has no conflicts of interest to declare. No funding was received for the publication of this article.

open Access: This article is published under the Creative Commons Attribution Noncommercial License, which permits any noncommercial use, distribution, adaptation, and reproduction provided the original author(s) and source are given appropriate credit.

Compliance with Ethical Guidance: Informed consent was received from the patient involved in this study.

Received: August 2, 2015 Accepted: August 17, 2015 Citation: US Ophthalmic Review, 2015;8(2):139-41 DOI: http://doi.org/10.17925/USOR.2015.08.02.139

Correspondence: Sudhir Singh, MS, Senior Consultant \& HOD, Department of Ophthalmology, JW Global Hospital \& Research Centre, Mount Abu, Rajasthan, India 307501.

E: drsudhirsingh@gmail.com

\section{Case Report}

A healthy 25-year-old female patient, with a 6-month history, reported with a chief complaint of left upper lid mass and droopiness. History revealed that the swelling initially started as a peanut size and had gradually increased to the present size. Ocular examination was suggestive of nontender, bony hard, noncompressible, nonfluctuant, and nonpulsatile swelling on the left upper lid (see Figure $1 A$ and $B$ ). The superior, inferior, medial, and lateral margins were well palpable. Mass was nonadherent to orbit, and it could be digitally displaced horizontally or vertically (see Figures $1 C$ and $D$ ). The size of the swelling was $25 \times 15 \times 10 \mathrm{~mm}$.

There was a history of blunt trauma to the left upper lid, and 3 months before she noticed this swelling. There was no history of vision changes, ocular pain, pain with eye movement, or headaches. Her vision was 20/20 in both the eyes. Both the eyes underwent slit lamp examination and fundus was also normal.

Past ocular history and medical history were noncontributory.

Review of systems was negative.

The patient was operated under local anesthesia. Two silk sutures were taken near the lid margin to provide traction during surgery. The globe was secured with an entropion plate. Lid crease incision was performed superficial to tumor (see Figures $2 A$ and $B$ ). The lesion was isolated from the surrounding tissue by blunt dissection. Dissection was easily carried out around the mass except for the lower portion, which was found adherent with tarsal plate (see Figures $2 B-D$ ). The tumor and its extent were identified. The lesion was excised completely (see Figure 2E). No communication with the orbit or sinuses could be identified.

The tarsal plate was sutured with 6-0 vicryl interrupted sutures. Skin was sutured with interrupted 6-0 vicryl sutures (see Figure 2F). Patient's ptosis was mostly recovered on 14th postoperative day (see Figure 3B). Specimens sent for histopathologic examination and histopathologic findings were consistent with an osteoid osteoma.

\section{Gross Examination}

The tumor was an encapsulated nodular lesion $25 \times 15 \times 10 \mathrm{~mm}$ in size. The consistency was bony hard. The anterior surface was glisteningwhite to pink color and was smooth with rounded protuberance. The cross-sections of specimen could not be cut by scalpel (see Figure 4).

\section{Microscopic Examination}

Histologic examination shows compact and mature bone and marrow space filled by connective tissue (see Figure 5).

\section{Discussion}

Osteoma is a benign slow-growing osteogenic lesion, composed of well-differentiated mature bone tissue, characterized by the proliferation 
Figure 1: A: Showing Severe Ptosis of the Left Upper Eyelid B: Showing Prominent Eyelid Swelling After Eye Closure C: Showing Downward Displacement of Lid Mass During Palpation D: Showing Lateral Displacement Of Lid Mass During Palpation
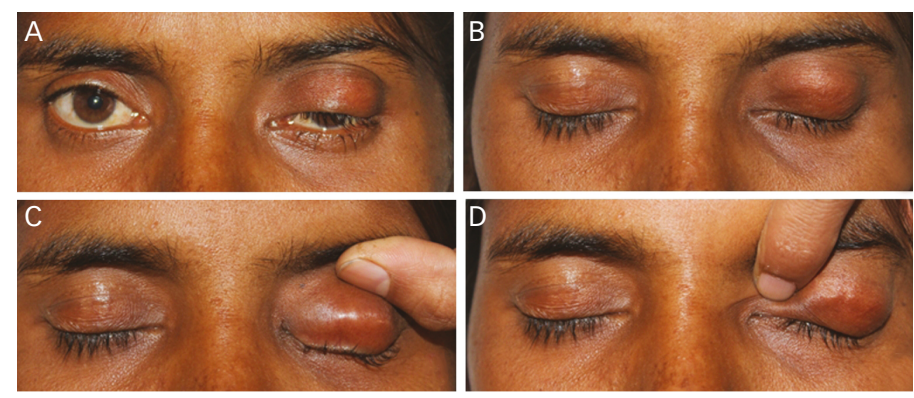

Figure 2: A: Muscle Deep Lid Crease Incision was Performed Superficial to Tumor B, C: Anterior Surface of the Eyelid Mass Exposed by Blunt Dissection D: Superior, Medial, and Lateral Margins were Dissected Out E: Status Postexcision of Eyelid Mass F: Appearance of the Eyelid after Wound Closure
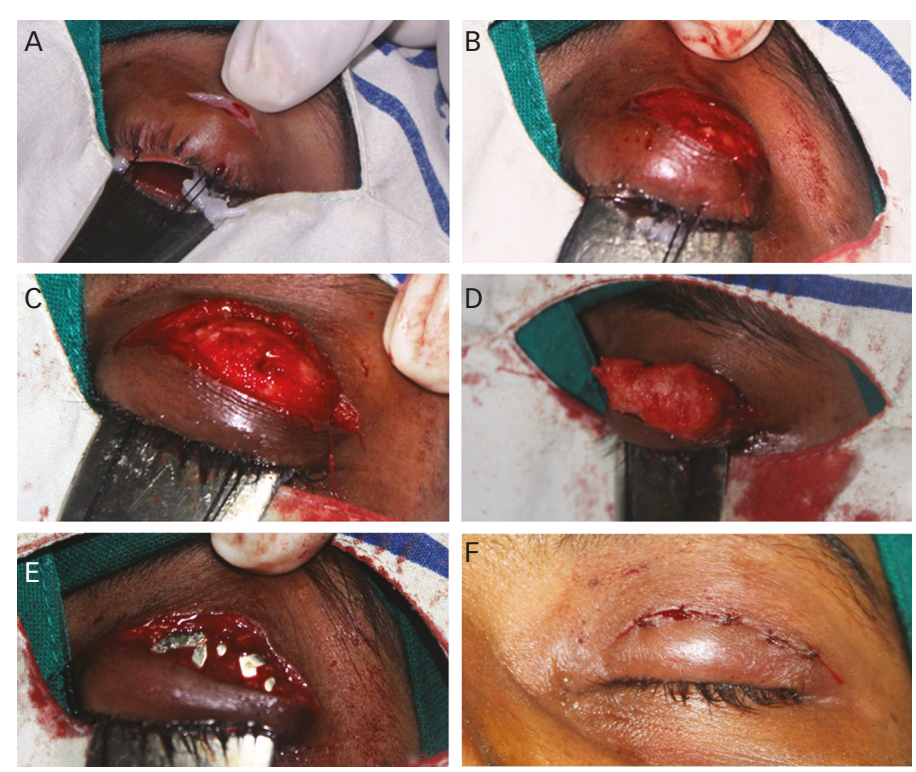

of compact or cancellous bone, almost exclusively found in the head and neck region. Central, peripheral, and extraskeletal are the three variants of osteoma. Trauma, inflammation, developmental disorders, and genetic defects are considered as the etiologic factors. ${ }^{1}$

Osteoma can occur at any age, but more frequently seen between the third and fifth decades. It has got a slight male predilection. ${ }^{2}$ Paranasal sinuses are the favorite locations of peripheral osteoma of the craniofacial region-frontal and ethmoidal sinuses being the common ones. External auditory canal, orbit, temporal bone, and pterygoid processes are the other locations. ${ }^{3}$ Primary orbital bone tumors constitute a very small percentage of all orbital tumors. More often, osteomas originate from the paranasal sinuses. Among the bony orbital tumors, fibrous dysplasias and osteomas are the most commonly encountered entities. ${ }^{4}$
Figure 3: A: Preoperative Patient's Photograph Showing Left Upper Eyelid Severe Ptosis and Lid Mass B: Two Weeks Postoperative Patient's Photograph Showing Correction of the Left Upper Eyelid Ptosis
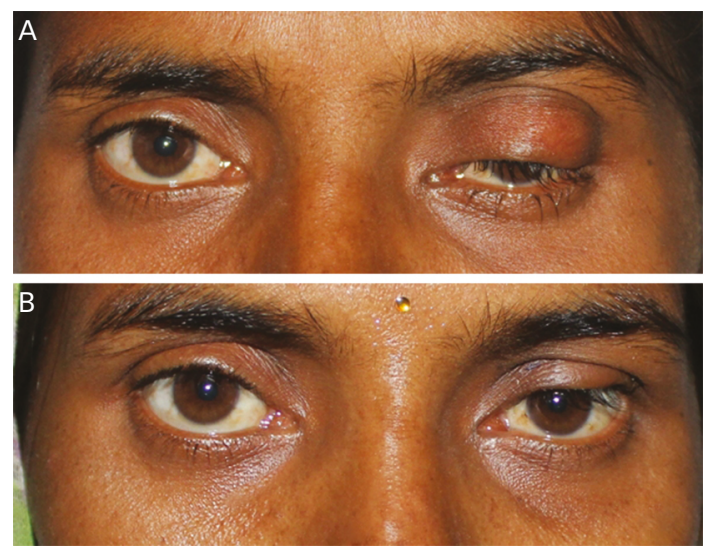

Figure 4: Showing Anterior and Posterior Gross Appearance of Extraskeletal Osteoma of Eyelid

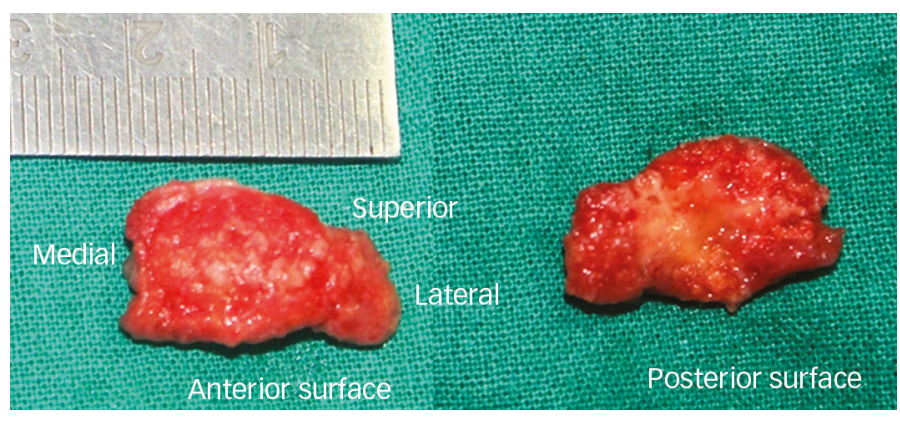

Figure 5: Histologic Examination Shows Compact and Mature Bone and Marrow Space Filled by Connective Tissue $(H \& E \times 10)$

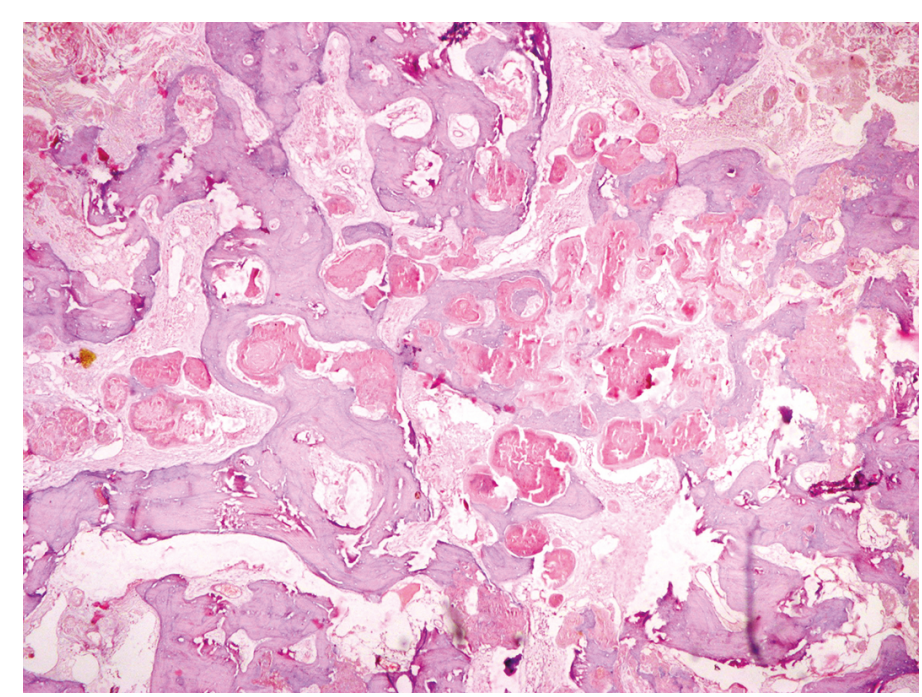

The case we reported here was an extraskeletal osteoma of the eyelid, as it was located in the left upper lid without any communication to orbit or 
paranasal sinuses. There was a history of blunt trauma to the left upper lid 3 months before this lid mass was noticed by the patient. The extraskeletal soft tissue osteoma usually develops within the muscle. In our case, extraskeletal osteoma probably was developed from orbicularis oculi of the left upper lid. During surgery, we found that this lesion was lying just beneath pretarsal muscle and anterior to tarsal palate. It was confined only to the eyelid. Extraskeletal osteoma of the lid is very rare. To the best of our knowledge, this is the first case of extraskeletal osteoma of the lid reported in the English language literature.

The osteoma is often slow growing and asymptomatic, diagnosed incidentally on radiographs. But later on, it can achieve a faster growth rate, as the rate of osteogenesis increases, and can cause deformation of the bone and compression of the adjacent structures. They usually appear as unilateral, sessile or pedunculated, well-circumscribed, mushroomlike masses, ranging from 1.5 to $40 \mathrm{~mm}$ in diameter. ${ }^{5}$ All these features are consistent with the present case. During physical examination, tumor was confined to eyelid and boundaries were well defined and palpable. So we did not order for computed tomography scan and planned this case for an excisional biopsy.

The differential diagnoses in this case include osteoid osteoma, dermoid cyst, and lipoma. Osteoma of the orbit can be seen as a bony hard swelling. ${ }^{6}$ Dermoid cyst and lipoma are also seen as subcutaneous nodules in the lid, but they will be soft and fluctuant. ${ }^{7}$

Management of osteoma of the eyelid is complete excision with clear margin to establish the histopathologic diagnosis and prevent recurrence.
1. Shanavas M, Chatra L, Shenai P, et al., Multiple peripheral osteomas of forehead: report of a rare case, Ann Med Health Sci Res, 2013;3:105-7.

2. Wanyura H, Kaminski A, Stopa Z, Treatment of osteomas located between the anterior cranial base and the face J Craniomaxillofac Surg, 2005;33:267-75.

3. Johann AC, de Freitas JB, de Aguiar MC, et al., Periphera osteoma of the mandible: case report and review of the literature, I Craniomaxillofac Surg, 2005;33:276-81

4. Selva D, White VA, O'Connell JX, Rootman J, Primary bone tumors of the orbit, Surv Ophthalmol 2004:49(3):328-42.

5. Kerckhaert A, Wolvius E, van der Wal K, Oosterhuisa JW, Giant osteoma of the mandible: case report, J Craniomaxillofac Surg, 2005;33:282-5

6. von Chamier G, Holl-Wieden A, Stenzel M, et al., Pitfalls in diagnostics of hip pain: osteoid osteoma and osteoblastoma, Rheumatol Int, 2010;30:395-400.

7. Sewell LD, Adams DC, Marks VJ, Subcutaneous forehead nodules: attention to the button osteoma and frontalis-associated lipoma, Dermatol Surg 2008;34:791-8

8. Sires BS, Benda PM, Stanley RB Jr, et al., Orbital osteoid osteoma, Arch Ophthalmol, 1999;117:414-5. 